\title{
Facile Access to Aldol Products from Aromatic and Heteroaromatic Aldehydes Using Ruthenium Catalyst
}

\author{
Khalil Tabatabaeian, Manouchehr Mamaghani, Nosrat O. Mahmoodi, and Elahe Keshavarz \\ Department of Chemistry, Faculty of Science, University of Guilan, P.O. Box 41335-1914, 4193833697, Rasht, Iran \\ Correspondence should be addressed to Khalil Tabatabaeian, taba@guilan.ac.ir
}

Received 2 November 2009; Accepted 22 February 2010

Academic Editor: Hakan Arslan

Copyright ( $) 2010$ Khalil Tabatabaeian et al. This is an open access article distributed under the Creative Commons Attribution License, which permits unrestricted use, distribution, and reproduction in any medium, provided the original work is properly cited.

A new method for the synthesis of aldol products is described. The reaction of aromatic and heteroaromatic aldehydes with 1(thiophen-2-yl)ethanone in the presence of ruthenium chloride hydrate combined with ligand provided the related aldol adducts, in a short time at room temperature in good yields (77-84\%).

\section{Introduction}

Lewis acid catalyzed reactions belong to one of the most powerful methods in modern synthetic chemistry. Among the many Lewis acids developed so far, ruthenium has received growing attention because of the unique combinations of their strong Lewis acidity mixed with mildness and usually high selectivity [1-3]. On the other hand, there has been great demand to develop one-pot procedures for successive reactions for the formation of several $\mathrm{C}-\mathrm{C}$ bonds. The aldol reaction is generally regarded as one of the most powerful and efficient $\mathrm{C}-\mathrm{C}$ bond forming reactions [4-6]. Aldol reactions can be catalyzed by bases. The reactions catalyzed by bases are not easily controlled, and the dehydration of the product is often unavoidable [7]. Organic molecules have also been used as the catalysts. However the byproduct from dehydration was still existed. Therefore, many efforts have been devoted to the development of catalytic aldol reactions [8-12].

In this research, cross-aldol reactions were carried out in a one-pot reaction by mixing the reaction components at room temperature. In the presence of catalytic quantities of $\mathrm{RuCl}_{3} \cdot n \mathrm{H}_{2} \mathrm{O}$ with triphenylphosphine, the reaction of 1-(thiophen-2-yl)ethanone with aldehydes resulted aldol adducts in good yields without the formation of any side product. Although a large number of catalytic and organocatalytic reactions exist, this work adds new knowledge to the field because to the best of our knowledge $\mathrm{Ru}^{\mathrm{III}}$ combined with ligand had never been used as a catalyst in catalytic cross aldol reactions of 1-(thiophen-2yl)ethanone with aromatic and heteroaromatic aldehydes.

\section{Results and Discussion}

As part of our continuing studies toward ruthenium catalyzed aldol reactions [13], it was found that $\mathrm{Ru}^{\mathrm{III}}$ in the presence of triphenylphosphine would be a more suitable catalyst for the mild formation of aldol adducts. In this method, the aldol products produced a shorter reaction times and simple experimental procedures. On the other hand, amount of $\mathrm{PPh}_{3}$ ligand used in this work was lower than when $\mathrm{Ru}^{\mathrm{II}}$ [14] was used. Here we report our new findings on the application of this catalytic system for the synthesis of aldol products at room temperature.

At the outset of this research, in order to optimize the reaction condition, the reaction of 1-(thiophen-2yl)ethanone 1 (3 mmol) and 4-(Methylthio)benzaldehyde 2a $(1 \mathrm{mmol})$ as model substrates, in the presence of ruthenium chloride hydrate in dioxane $(1 \mathrm{~mL})$ at room temperature was carried out and the effect of $\mathrm{PPh}_{3}$ to $\mathrm{Ru}$ mole ratio on the aldolization was studied (Table 1). Among the catalytic systems examined, commercial ruthenium trichloride hydrate was an efficient catalyst (Table 1, entry 1), but the yield 
TABLE 1: Effect of the molar ratio of $\mathrm{PPh}_{3}$ to $\mathrm{RuCl}_{3} \cdot n \mathrm{H}_{2} \mathrm{O}$ on the synthesis of aldol products.

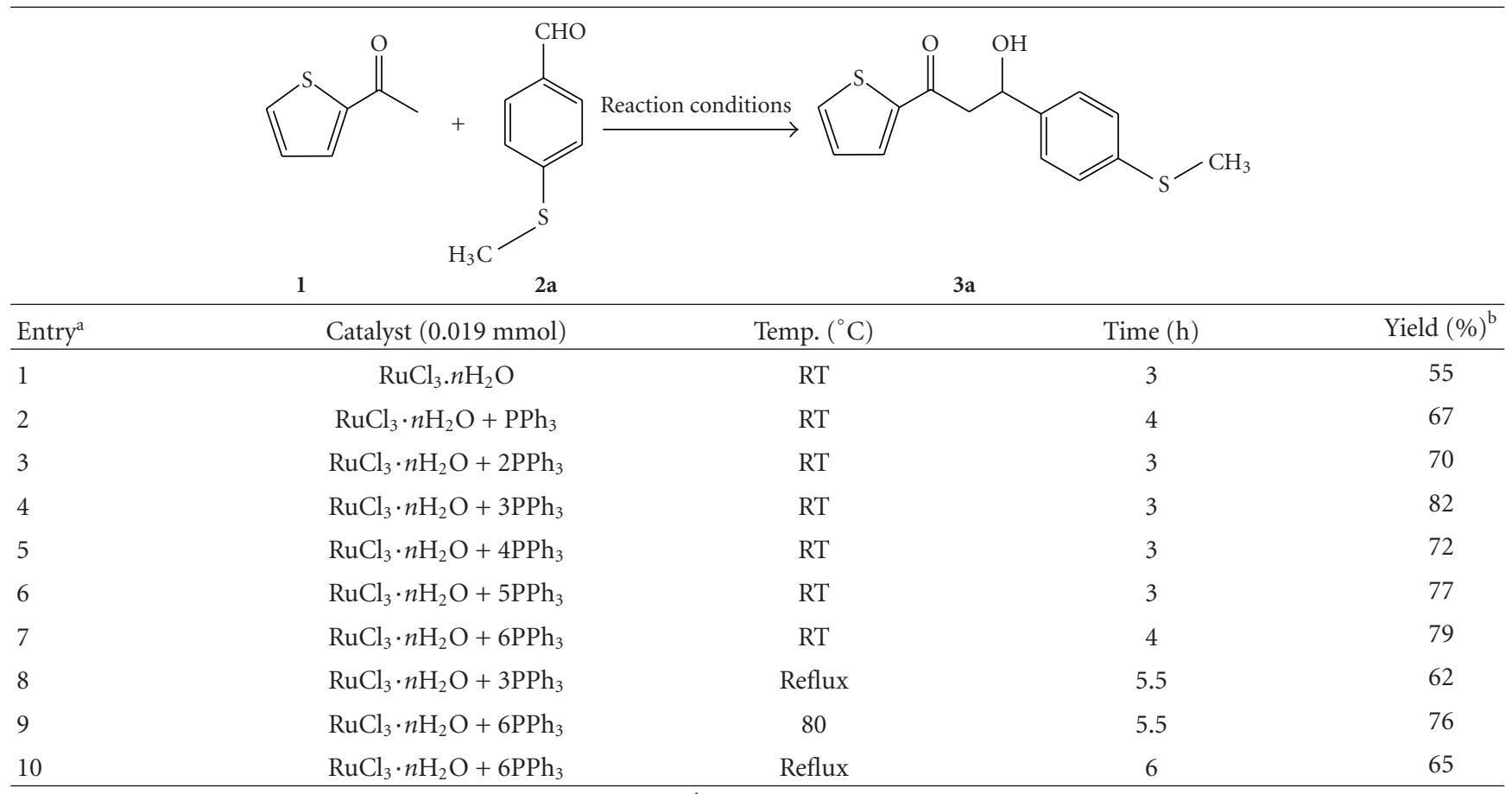

${ }^{a}$ All products were characterized by ${ }^{1} \mathrm{H}$ NMR, ${ }^{13} \mathrm{C}$ NMR, and IR data. ${ }^{\text {b}}$ Yields after purification by chromatography.<smiles>CC(=O)c1cccs1</smiles>

1<smiles>[R]C=O</smiles>

$2 a-\mathbf{i}$

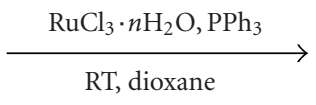

RT, dioxane

$\mathrm{R}: 4-\mathrm{MeSC}_{6} \mathrm{H}_{4}, 3$-methylthiophen-2-yl, 5-methylthiophen-2-yl, thiophen-2-yl, furan-2-yl, 4- $\mathrm{MeOC}_{6} \mathrm{H}_{4}, 4-\mathrm{MeC}_{6} \mathrm{H}_{4}, 2-\mathrm{MeOC}_{6} \mathrm{H}_{4}, \mathrm{C}_{6} \mathrm{H}_{5}$<smiles>[R]C(O)CC(=O)c1cccs1</smiles>

$3 a-i$

Scheme 1: Ruthenium-catalyzed cross-aldol reaction using $\mathrm{RuCl}_{3} \cdot n \mathrm{H}_{2} \mathrm{O} / 3 \mathrm{PPh}_{3}(3 \mathbf{a}-\mathbf{i})$.

of $3 \mathbf{a}$ could be greatly increased by adding $\mathrm{PPh}_{3}$. Addition of an equal amount of $\mathrm{PPh}_{3}$ ligand with the $\mathrm{RuCl}_{3} \cdot n \mathrm{H}_{2} \mathrm{O}$ enhanced the catalytic activity to give $3 \mathbf{a}$ in yield of $67 \%$ (Table 1, entry 2). Moreover, when the amount of $\mathrm{PPh}_{3}$ was increased to $0.057 \mathrm{mmol}$ (Table 1, entry 4), the formation of the $\mathbf{3 a}$ became the predominant product and no byproduct was detected. On the other hand, more addition of $\mathrm{PPh}_{3}$ ligand did not improve the reaction. Thus, $\mathrm{PPh}_{3}$ to $\mathrm{Ru}$ ratio was kept at 3 in the following experiments.

Therefore, when aldehydes ( $1 \mathrm{mmol}), \mathrm{RuCl}_{3} \cdot n \mathrm{H}_{2} \mathrm{O}$ $(0.019 \mathrm{mmol}), \mathrm{PPh}_{3}(0.057 \mathrm{mmol})$, and 1-(thiophen-2yl)ethanone $(3 \mathrm{mmol})$ were added at room temperature in dioxane $(1 \mathrm{~mL})$ (Scheme 1$)$, the aldol products $3 \mathbf{a}-\mathbf{i}$ were generated within (3-4 hours) (Table 2).

Considering the results described above, the most plausible mechanism is illustrated in Scheme 2. The mechanism involves activation of the substrates within the coordination sphere of the metal.

\section{Conclusions}

Prompted by our findings and intrigued by diverse reactivities of ruthenium compounds [15-18], we have found that the coupling of aldehydes with 1-(thiophen-2-yl)ethanone is achieved in a short time under the influence of $\mathrm{RuCl}_{3} \cdot n \mathrm{H}_{2} \mathrm{O}$ combined with $\mathrm{PPh}_{3}$ to give aldol products in good yields. We were pleased to find that catalytic amounts of ruthenium at room temperature cleanly produced aldol products. More importantly, there was no side product produced and the estimated molar waste was small.

\section{Experimental}

4.1. General Methods. All solvents, organic, and inorganic compounds were purchased from Merck and used without further purification. All reactions were followed by TLC with detection by UV light. 
TABLE 2: Ruthenium-catalyzed cross-aldol reaction in the presence of $\mathrm{RuCl} 3 \cdot n \mathrm{H} 2 \mathrm{O} / 3 \mathrm{PPh} 3(3 \mathrm{a}-\mathbf{i})$.

\begin{tabular}{|c|c|c|c|c|}
\hline Entry $^{\mathrm{a}}$ & Aldehyde & Product & Time $(\mathrm{h})$ & Yield $(\%)^{\mathrm{b}}$ \\
\hline 1 & 4-(Methylthio)benzaldehyde & $3 a$ & 3 & 82 \\
\hline 2 & 3-Methylthiophen-2-carbaldehyde & $3 b$ & 3.5 & 78 \\
\hline 3 & 5-Methylthiophen-2-carbaldehyde & $3 c$ & 4 & 80 \\
\hline 4 & Thiophen-2-carbaldehyde & $3 d$ & 3.5 & 84 \\
\hline 5 & Furan-2-carbaldehyde & $3 e$ & 3.5 & 77 \\
\hline 6 & 4-Methoxybenzaldehyde & $3 f$ & 3 & 80 \\
\hline 7 & 4-Methylybenzaldehyde & $3 g$ & 3.5 & 81 \\
\hline 8 & 2-Methoxybenzaldehyde & $3 h$ & 3.5 & 77 \\
\hline 9 & Benzaldehyde & $3 \mathbf{i}$ & 3 & 83 \\
\hline
\end{tabular}

${ }^{\mathrm{a}}$ All products were characterized by ${ }^{1} \mathrm{H} \mathrm{NMR},{ }^{13} \mathrm{C}$ NMR, and IR data. ${ }^{\mathrm{b}}$ Yields after purification by chromatography.

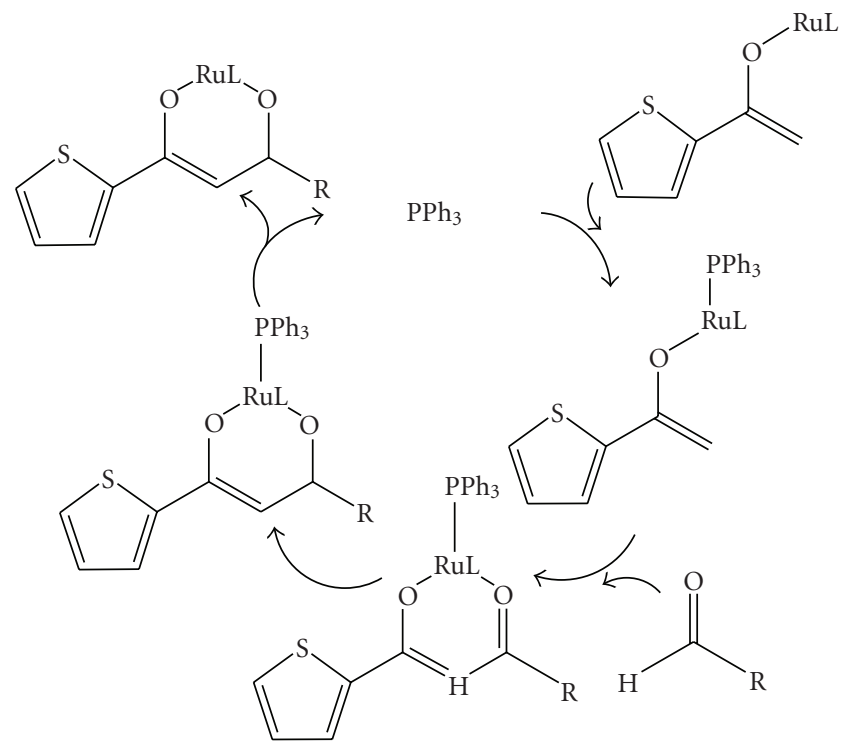

Scheme 2: Proposed mechanism for ruthenium-catalyzed aldol reactions.

IR spectra were recorded on Shimadzu FTIR-8400S spectrometer. ${ }^{1} \mathrm{H}$ NMR spectra were obtained on a Bruker DRX-500 Avance spectrometer, and ${ }^{13} \mathrm{C}$ NMR were obtained on a Bruker DRX-125 Avance spectrometer. Samples were analyzed in $\mathrm{CDCl}_{3}$, and the chemical shift values are reported in ppm relative to (tetramethylsilane) TMS as the internal reference. Elemental analyses were made by a Carlo-Erba EA1110 CHNO-S analyzer and agreed with the calculated values. The isolation of pure products was carried out via preparative thin layer chromatography (silica gel $60 \mathrm{GF}_{254}$, Merck).

Excess of solvent was evaporated under reduced pressure at a bath temperature of 50 and $60^{\circ} \mathrm{C}$.

4.2. Typical Experimental Procedure for Synthesis of Aldol Products (3a-i). A mixture of aldehyde (1 mmol), 1(thiophen-2-yl)ethanone $(3 \mathrm{mmol}), \mathrm{RuCl}_{3} \cdot n \mathrm{H}_{2} \mathrm{O}$ (4 mg, $0.019 \mathrm{mmol})$, and $\mathrm{PPh}_{3}(15 \mathrm{mg}, 0.057 \mathrm{mmol})$ in dioxane $(1 \mathrm{~mL})$ was stirred at room temperature and monitored by TLC. After the indicated reaction time (Table 2), the reaction mixture was purified by thin layer chromatography (EtOAc/petroleum ether $1: 4 \mathrm{v} / \mathrm{v})$, providing the aldol adduct. In this method, no other products were observed.

3-Hydroxy-3-(4-(methylthio)phenyl)-1-(thiophen-2yl)propan-1-one (3a): yellow oil, IR (neat): 3400, 2920, 1700, 1645, $1465 \mathrm{~cm}^{-1} .{ }^{1} \mathrm{H}$ NMR $\left(500 \mathrm{MHz}, \mathrm{CDCl}_{3}\right): \delta$ $2.53(\mathrm{~m}, 3 \mathrm{H}), 3.30(\mathrm{dd}, J=16.0,6.9 \mathrm{~Hz}, 1 \mathrm{H}), 3.43(\mathrm{dd}$, $J=16.0,6.9 \mathrm{~Hz}, 1 \mathrm{H}), 3.57(\mathrm{~d}, J=3.0 \mathrm{~Hz}, \mathrm{OH}), 5.33(\mathrm{~m}$, 1H), 7.41-7.14 (m, 4H), 7.79-7.65 (m, 3H) ppm. ${ }^{13} \mathrm{C} \mathrm{NMR}$ $\left(125 \mathrm{MHz}, \mathrm{CDCl}_{3}\right): \delta 16.4,45.7,70.3,127.3,127.5,128.4$, $128.6,132.6,133.1,134.2,144.6$ and 214.2 ppm. (Found: C, 60.42; $\mathrm{H}, 5.07 ; \mathrm{S}, 23.03$. Calc. for $\mathrm{C}_{14} \mathrm{H}_{14} \mathrm{O}_{2} \mathrm{~S}_{2}$ : C, 60.43; $\mathrm{H}$, $5.03 ;$ S, $23.02 \%$.)

3-Hydroxy-3-(3-methylthiophen-2-yl)-1-(thiophen-2yl)propan-1-one (3b): yellow oil, IR (neat): 3578, 3118, 2965, $1674,1564,1465 \mathrm{~cm}^{-1} .{ }^{1} \mathrm{H}$ NMR $\left(500 \mathrm{MHz}, \mathrm{CDCl}_{3}\right): \delta 2.40$ $(\mathrm{s}, 3 \mathrm{H}), 3.45(\mathrm{~m}, 2 \mathrm{H}), 4.01(\mathrm{~d}, J=3.4 \mathrm{~Hz}, \mathrm{OH}), 5.30(\mathrm{~m}$, $1 \mathrm{H}), 6.70(\mathrm{~d}, J=3.3 \mathrm{~Hz}, 1 \mathrm{H}), 6.87(\mathrm{~d}, J=3.3 \mathrm{~Hz}, 1 \mathrm{H}), 7.21$ $(\mathrm{m}, 1 \mathrm{H}), 7.72(\mathrm{~d}, J=4.8 \mathrm{~Hz}, 1 \mathrm{H})$ and $7.81(\mathrm{~d}, J=3.7 \mathrm{~Hz}$, $1 \mathrm{H}) \mathrm{ppm} .{ }^{13} \mathrm{C} \mathrm{NMR}\left(125 \mathrm{MHz}, \mathrm{CDCl}_{3}\right): \delta 15.9,52.4,67.5$, $125.3,125.9,128.6,134.7,135.5,138.6,141.7,142.0$ and 192.1 ppm. (Found: C, 57.16; H, 4.80; S, 25.40. Calc. for $\mathrm{C}_{12} \mathrm{H}_{12} \mathrm{O}_{2} \mathrm{~S}_{2}$ : C, 57.14; H, 4.76; S, 25.39\%.)

3-Hydroxy-3-(5-methylthiophen-2-yl)-1-(thiophen-2yl)propan-1-one (3c): yellow oil, IR (neat): 3589, 3088, 2960, $1675,1575,1466 \mathrm{~cm}^{-1} .{ }^{1} \mathrm{H}$ NMR $\left(500 \mathrm{MHz}, \mathrm{CDCl}_{3}\right): \delta 2.51$ $(\mathrm{s}, 3 \mathrm{H}), 3.48(\mathrm{~m}, 2 \mathrm{H}), 3.59(\mathrm{~d}, J=3.5 \mathrm{~Hz}, \mathrm{OH}), 5.50(\mathrm{~m}$, $1 \mathrm{H}), 6.66(\mathrm{~d}, J=3.3 \mathrm{~Hz}, 1 \mathrm{H}), 6.86(\mathrm{~d}, J=3.3 \mathrm{~Hz}, 1 \mathrm{H}), 7.20$ $(\mathrm{m}, 1 \mathrm{H}), 7.74(\mathrm{~d}, J=4.9 \mathrm{~Hz}, 1 \mathrm{H})$ and $7.79(\mathrm{~d}, J=3.7 \mathrm{~Hz}$, $1 \mathrm{H})$ ppm. ${ }^{13} \mathrm{C} \mathrm{NMR}\left(125 \mathrm{MHz}, \mathrm{CDCl}_{3}\right): \delta 15.7,50.2,68.9$, $125.6,126.4,128.4,133.3,134.5,138.5,141.5,141.8$ and 192.0 ppm. (Found: C, 57.18; H, 4.80; S, 25.41. Calc. for $\mathrm{C}_{12} \mathrm{H}_{12} \mathrm{O}_{2} \mathrm{~S}_{2}$ : C, 57.14; H, 4.76; S, 25.39\%.)

3-Hydroxy-1,3-di(thiophen-2-yl)propan-1-one (3d): yellow oil, IR (neat): 3400, 3095, 2925, 1651, 1601, 1515, $1413 \mathrm{~cm}^{-1} .{ }^{1} \mathrm{H}$ NMR $\left(500 \mathrm{MHz}, \mathrm{CDCl}_{3}\right): \delta 3.41$ (dd, $J=16.0$, $6.0 \mathrm{~Hz}, 1 \mathrm{H}), 3.53(\mathrm{dd}, J=16.0,6.0 \mathrm{~Hz}, 1 \mathrm{H}), 3.54(\mathrm{~d}, J=$ $3.5 \mathrm{~Hz}, \mathrm{OH}), 4.45(\mathrm{~m}, 1 \mathrm{H}), 6.95-6.91(\mathrm{~m}, 2 \mathrm{H}), 7.17(\mathrm{~m}, 2 \mathrm{H})$, $7.67(\mathrm{~m}, 1 \mathrm{H})$ and $7.81(\mathrm{~m}, 1 \mathrm{H}) \mathrm{ppm} .{ }^{13} \mathrm{C} \mathrm{NMR}(125 \mathrm{MHz}$, $\left.\mathrm{CDCl}_{3}\right): \delta 50.2,69.0,125.4,126.1,126.6,128.6,133.3,134.5$, 141.9, 142.9 and $192.2 \mathrm{ppm}$. (Found: C, 55.50; H, 4.24; S, 26.92. Calc. for $\mathrm{C}_{11} \mathrm{H}_{10} \mathrm{O}_{2} \mathrm{~S}_{2}$ : C, 55.46; $\mathrm{H}, 4.20$; $\mathrm{S}, 26.89 \%$.) 
3-(Furan-2-yl)-3-hydroxy-1-(thiophen-2-yl)propan-1one (3e): yellow oil, IR (neat): 3400, 3382, 2925, 1656, 1517, $1465 \mathrm{~cm}^{-1}$. ${ }^{1} \mathrm{H}$ NMR $\left(500 \mathrm{MHz}, \mathrm{CDCl}_{3}\right): \delta 3.40(\mathrm{dd}, J=$ $16.0,6.0 \mathrm{~Hz}, 1 \mathrm{H}), 3.47(\mathrm{dd}, J=16.0,6.0 \mathrm{~Hz}, 1 \mathrm{H}), 3.70(\mathrm{~d}, J$ $=3.5 \mathrm{~Hz}, \mathrm{OH}), 4.21(\mathrm{~m}, 1 \mathrm{H}), 6.12(\mathrm{~m}, 1 \mathrm{H}), 6.27(\mathrm{~m}, 1 \mathrm{H})$, 7.32-7.15 (m, 2H), $7.67(\mathrm{~m}, 1 \mathrm{H})$ and $7.82(\mathrm{~m}, 1 \mathrm{H}) \mathrm{ppm} .{ }^{13} \mathrm{C}$ $\mathrm{NMR}\left(125 \mathrm{MHz}, \mathrm{CDCl}_{3}\right): \delta 47.5,63.2,105.8,110.0,128.5$, 133.3, 134.2, 141.5, 141.8, 152.8 and 192.2 ppm. (Found: C, 59.43; $\mathrm{H}, 4.54 ; \mathrm{S}, 14.43$. Calc. for $\mathrm{C}_{11} \mathrm{H}_{10} \mathrm{O}_{3} \mathrm{~S}$ : C, 59.45; $\mathrm{H}$, $4.50 ; \mathrm{S}, 14.41 \%$.

3-Hydroxy-3-(4-methoxyphenyl)-1-(thiophen-2-yl)propan-1-one (3f): yellow oil, IR (neat): 3450, 2925, 1700, 1635, $1455 \mathrm{~cm}^{-1} .{ }^{1} \mathrm{H}$ NMR $\left(500 \mathrm{MHz}, \mathrm{CDCl}_{3}\right): \delta 3.55(\mathrm{~m}, 3 \mathrm{H})$, $3.33(\mathrm{dd}, J=16.0,6.8 \mathrm{~Hz}, 1 \mathrm{H}), 3.40(\mathrm{dd}, J=16.0,6.8 \mathrm{~Hz}$, $1 \mathrm{H}), 3.56(\mathrm{~d}, J=3.1 \mathrm{~Hz}, \mathrm{OH}), 5.38(\mathrm{~m}, 1 \mathrm{H}), 7.45-7.15(\mathrm{~m}$, $4 \mathrm{H}), 7.83-7.67(\mathrm{~m}, 3 \mathrm{H}) \mathrm{ppm} .{ }^{13} \mathrm{C} \mathrm{NMR}\left(125 \mathrm{MHz}, \mathrm{CDCl}_{3}\right)$ : $\delta$ 42.7, 50.3, 70.3, 117.3, 117.5, 128.6, 128.7, 132.6, 133.1, 134.2, 154.6 and $214.1 \mathrm{ppm}$. (Found: C, 64.14; H, 5.31; S, 12.25. Calc. for $\mathrm{C}_{14} \mathrm{H}_{14} \mathrm{O}_{3} \mathrm{~S}$ : C, 64.12; $\mathrm{H}, 5.34 ; \mathrm{S}, 12.21 \%$.)

3-Hydroxy-1-(thiophen-2-yl)-3-p-tolylpropan-1-one (3g): yellow oil, IR (neat): $3450,2925,1700,1635,1455 \mathrm{~cm}^{-1} .{ }^{1} \mathrm{H}$ $\mathrm{NMR}\left(500 \mathrm{MHz}, \mathrm{CDCl}_{3}\right): \delta 3.50(\mathrm{~m}, 3 \mathrm{H}), 3.30(\mathrm{dd}, J=$ 16.0, $6.8 \mathrm{~Hz}, 1 \mathrm{H}), 3.49(\mathrm{dd}, J=16.0,6.8 \mathrm{~Hz}, 1 \mathrm{H}), 2.50(\mathrm{~s}$, $\mathrm{OH}), 5.29(\mathrm{~m}, 1 \mathrm{H}), 7.41-7.25(\mathrm{~m}, 4 \mathrm{H}), 7.80-7.66(\mathrm{~m}, 3 \mathrm{H})$ ppm. ${ }^{13} \mathrm{C} \mathrm{NMR}\left(125 \mathrm{MHz}, \mathrm{CDCl}_{3}\right): \delta 42.7,50.3,70.3,117.3$, $117.5,128.6,128.7,132.6,133.1,134.2,154.6$ and $214.1 \mathrm{ppm}$. (Found: C, 68.28; H, 5.73; S, 13.02. Calc. for $\mathrm{C}_{14} \mathrm{H}_{14} \mathrm{O}_{2} \mathrm{~S}$ : C, 68.29; H, 5.69; S, 13.00\%.)

3-Hydroxy-3-(2-methoxyphenyl)-1-(thiophen-2-yl)propan-1-one (3h): yellow oil, IR (neat): 3452, 2935, 1700, 1615, $1445 \mathrm{~cm}^{-1} .{ }^{1} \mathrm{H}$ NMR $\left(500 \mathrm{MHz}, \mathrm{CDCl}_{3}\right): \delta 3.55(\mathrm{~m}, 3 \mathrm{H})$, $3.31(\mathrm{dd}, J=16.0,6.8 \mathrm{~Hz}, 1 \mathrm{H}), 3.44(\mathrm{dd}, J=16.0,6.8 \mathrm{~Hz}$, $1 \mathrm{H}), 3.54(\mathrm{~d}, J=3.2 \mathrm{~Hz}, \mathrm{OH}), 5.35(\mathrm{~m}, 1 \mathrm{H}), 7.46-7.12(\mathrm{~m}$, $4 \mathrm{H}), 7.81-7.69(\mathrm{~m}, 3 \mathrm{H}) \mathrm{ppm} .{ }^{13} \mathrm{C} \mathrm{NMR}\left(125 \mathrm{MHz}, \mathrm{CDCl}_{3}\right)$ : $\delta$ 42.9, 55.3, 72.3, 117.1, 117.4, 128.6, 128.7, 132.6, 133.3, 134.2, 154.5 and 214.2 ppm. (Found: C, 64.11; H, 5.32; S, 12.23. Calc. for $\mathrm{C}_{14} \mathrm{H}_{14} \mathrm{O}_{3} \mathrm{~S}$ : C, 64.12; H, 5.34; S, 12.21\%.)

3-Hydroxy-3-phenyl-1-(thiophen-2-yl)propan-1-one (3i): yellow oil, IR (neat): $3460,2950,1700,1625,1475 \mathrm{~cm}^{-1} \cdot{ }^{1} \mathrm{H}$ $\mathrm{NMR}\left(500 \mathrm{MHz}, \mathrm{CDCl}_{3}\right): \delta 3.35(\mathrm{dd}, J=16.0,6.8 \mathrm{~Hz}, 1 \mathrm{H})$, $3.45(\mathrm{dd}, J=16.0,6.8 \mathrm{~Hz}, 1 \mathrm{H}), 2.50(\mathrm{~s}, \mathrm{OH}), 4.80(\mathrm{~m}$, 1H), 7.25-7.19 (m, 5H), 7.59-7.20 (m, 3H) ppm. ${ }^{13} \mathrm{C}$ NMR $\left(125 \mathrm{MHz} \mathrm{CDCl}_{3}\right): \delta 42.9,72.3,117.1,117.4,128.6,128.7$, 132.6, 133.3, 134.2, 154.5 and 214.2 ppm. (Found: C, 67.28; $\mathrm{H}, 5.18 ; \mathrm{S}, 13.80$. Calc. for $\mathrm{C}_{13} \mathrm{H}_{12} \mathrm{O}_{2} \mathrm{~S}$ : C, 67.24; H, 5.17; S, $13.79 \%$.)

\section{Acknowledgment}

The authors are grateful to the Research Council of Guilan University for the support of this study.

\section{References}

[1] S.-I. Murahashi, Ruthenium in Organic Synthesis, Wiley-VCH, New York, NY, USA, 2004.

[2] C. S. Yi, S. Y. Yun, and Z. He, "Conjugate addition of alcohols to acrylic compounds catalyzed by a bifunctional ruthenium- acetamido complex," Organometallics, vol. 22, no. 15, pp. 3031-3033, 2003.

[3] P.-B. Ilan and B. Ouri, "Transformation of carbinols by $\mathrm{RuCl}_{2}\left(\mathrm{PPh}_{3}\right)_{3}$ and by some other transition-metal catalysts," The Journal of Organic Chemistry, vol. 45, pp. 4418-4428, 1980.

[4] T. Mukaiyama, "Explorations into new reaction chemistry," Angewandte Chemie International Edition, vol. 43, no. 42, pp. 5590-5614, 2004.

[5] T. D. Machajewski and C.-H. Wong, "The catalytic asymmetric aldol reaction," Angewandte Chemie International Edition, vol. 39, no. 8, pp. 1352-1374, 2000.

[6] R. Mahrwald, "Diastereoselection in Lewis-acid-mediated aldol additions," Chemical Reviews, vol. 99, no. 5, pp. 10951120, 1999.

[7] J. March, Advanced Organic Chemistry: Reactions, Mechanisms and Structure, Wiley-Interscience, New York, NY, USA, 4th edition, 1992.

[8] H.-X. Wei, R. L. Jasoni, H. Shao, J. Hu, and P. W. Paré, "Antiselective and regioselective aldol addition of ketones with aldehydes using $\mathrm{MgI}_{2}$ as promoter," Tetrahedron, vol. 60, no. 51, pp. 11829-11835, 2004.

[9] M. Wang and C.-J. Li, "Aldol reaction via in situ olefin migration in water," Tetrahedron Letters, vol. 43, no. 19, pp. 3589-3591, 2002.

[10] S. Matsunaga, T. Ohshima, and M. Shibasaki, "LinkedBINOL: an approach towards practical asymmetric multifunctional catalysis," Advanced Synthesis \& Catalysis, vol. 344, no. 1, pp. 3-15, 2002.

[11] B. M. Trost, E. R. Silcoff, and H. Ito, "Direct asymmetric aldol reactions of acetone using bimetallic zinc catalysts," Organic Letters, vol. 3, no. 16, pp. 2497-2500, 2001.

[12] N. Yoshikawa, N. Kumagai, S. Matsunaga, et al., "Direct catalytic asymmetric aldol reaction: synthesis of either syn-or anti- $\alpha, \beta$-dihydroxy ketones," Journal of the American Chemical Society, vol. 123, no. 10, pp. 2466-2467, 2001.

[13] K. Tabatabaeian, M. Mamaghani, N. O. Mahmoodi, and E. Keshavarz, "Ruthenium-catalyzed cross aldol reaction with aldehydes and ketones," Arkivoc, vol. 2009, no. 2, pp. 68-75, 2009.

[14] T. A. Stephenson and G. Wilkinson, "New complexes of ruthenium (II) and (III) with triphenylphosphine, triphenylarsine, trichlorostannate, pyridine and other ligands," Journal of Inorganic and Nuclear Chemistry, vol. 28, no. 4, pp. 945-956, 1966.

[15] K. Tabatabaeian, M. Mamaghani, N. O. Mahmoodi, and A. Khorshidi, "Ultrasonic-assisted ruthenium-catalyzed oxidation of aromatic and heteroaromatic compounds," Catalysis Communications, vol. 9, no. 3, pp. 416-420, 2008.

[16] K. Tabatabaeian, M. Mamaghani, N. O. Mahmoodi, and A. Khorshidi, " $\mathrm{Ru}^{I I I}$-catalyzed double-conjugate 1,4-addition of indoles to symmetric enones," Journal of Molecular Catalysis A, vol. 270, no. 1-2, pp. 112-116, 2007.

[17] K. Tabatabaeian, M. Mamaghani, N. Mahmoodi, and A. Khorshidi, "Efficient $\mathrm{Ru}^{I I I}$-catalyzed condensation of indoles and aldehydes or ketones," Canadian Journal of Chemistry, vol. 84, no. 11, pp. 1541-1545, 2006.

[18] K. Tabatabaeian, M. Mamaghani, N. O. Mahmoodi, and A. Khorshidi, "Solvent-free, ruthenium-catalyzed, regioselective ring-opening of epoxides, an efficient route to various 3alkylated indoles," Tetrahedron Letters, vol. 49, pp. 1450-1454, 2008. 


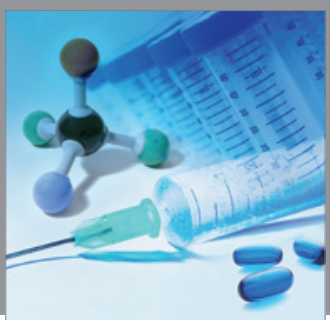

International Journal of

Medicinal Chemistry

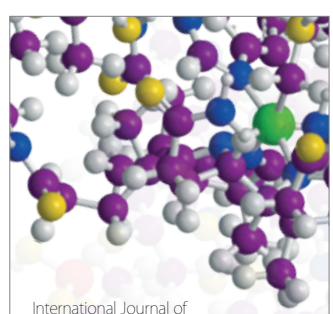

Carbohydrate Chemistry

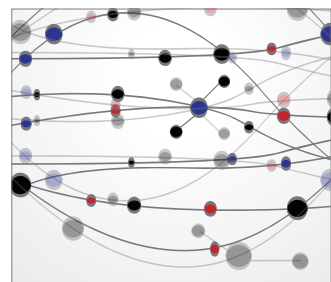

The Scientific World Journal
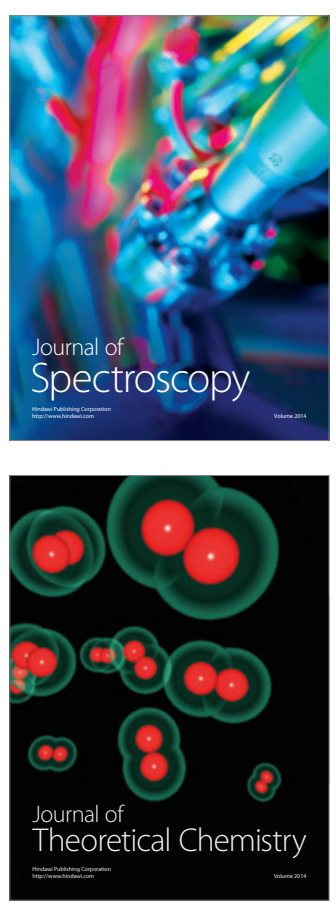
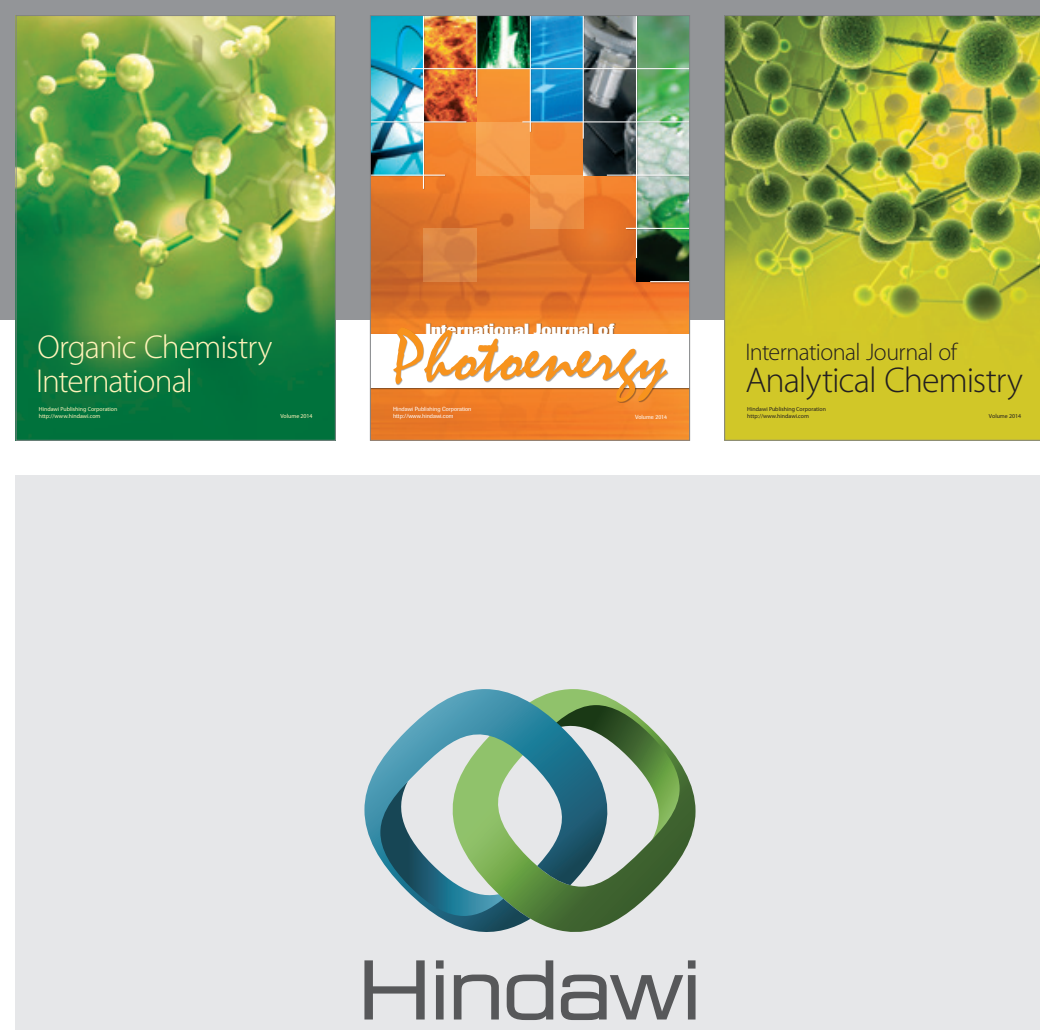

Submit your manuscripts at

http://www.hindawi.com
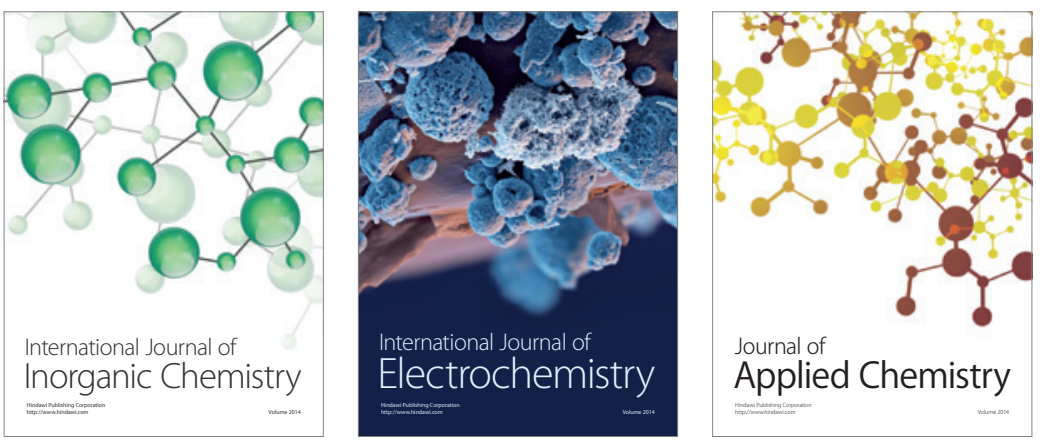

Journal of

Applied Chemistry
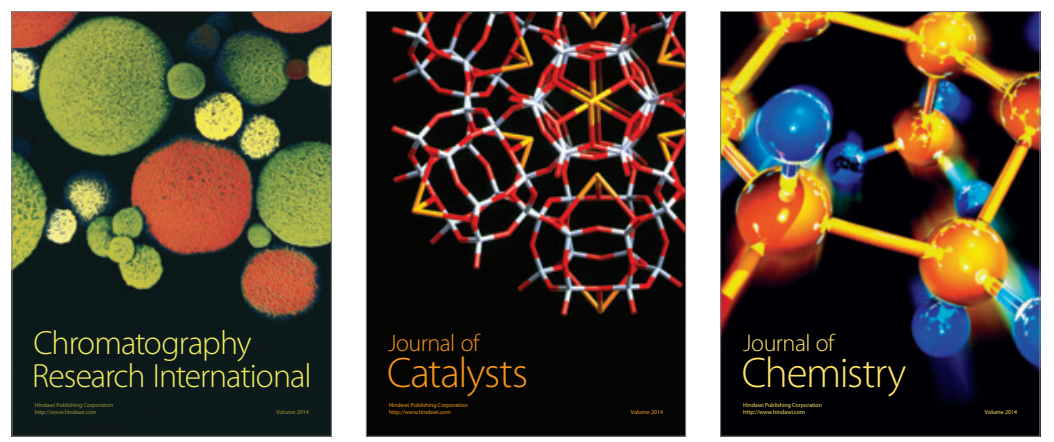
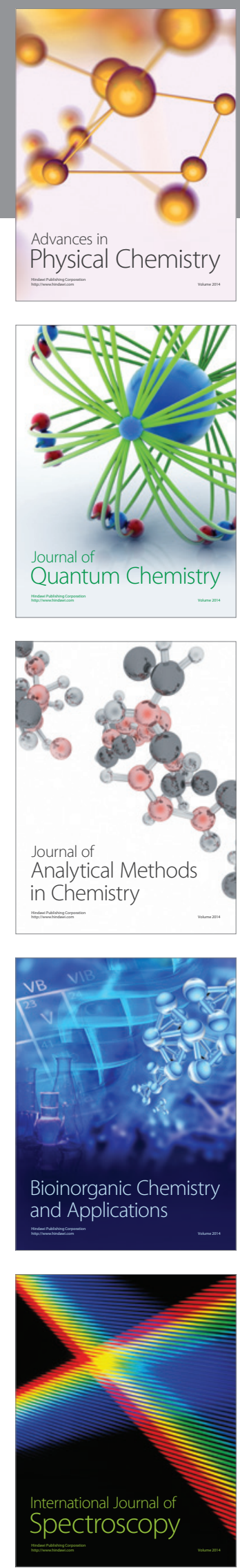